\title{
Effet signal d'une réduction promotionnelle du prix : concept et expérimentation
}

\author{
Pierre Desmet
}

RÉS U MÉ

L'effet observé à court terme d'une réduction promotionnelle du prix sur la demande est souvent positif du fait de la valorisation des bénéfices offerts par la promotion et par le simple effet signal engendré par la communication promotionnelle.

Lorsque le consommateur effectue une évaluation approfondie de l'offre promotionnelle et qu'il manque des informations (sur le montant de la réduction ou le prix de base), l'information transmise par la promotion, qui est à la source de l'effet signal, est aussi utilisée pour élaborer le cadre du choix. Un conflit entre la cherté perçue et le signal promotionnel peut donc dévaloriser une offre.

Dans ces conditions, nous montrons, par l'analyse des résultats d'une expérimentation sur 320 consommateurs, que le changement du cadre du choix résultant d'une offre promotionnelle conduit à un rejet d'achat plus important d'une marque premium pour un produit de grande consommation.

Si ce résultat d'un effet négatif d'une réduction promotionnelle du prix sur la demande à court terme est rarement observé sur les ventes, il doit cependant conduire les managers à anticiper une baisse de l'efficacité promotionnelle lorsque la réduction de prix n'est pas indiquée et à en limiter l'usage pour les marques premium.

Mots clés : Promotion des ventes, réduction de prix, effet signal, expérimentation, Gabor et Granger.

\section{INTRODUCTION}

Les techniques de prix sont les techniques promotionnelles les plus utilisées pour les produits de grande consommation (63\% en 2002, selon la pige BIPP). Elles regroupent des techniques de réduction immédiate ou différée du prix, des bons de réduction et escomptes, et des offres de remboursement. Lorsqu'elles sont mises en œuvre par un fournisseur, les techniques de réduction immédiate ne permettent pas à

L'auteur peut être contacté à l'adresse électronique suivante : desmet@ dauphine.fr

L' auteur tient à remercier Monsieur Dedeyan, de la société IOD, pour son soutien et pour la collecte des données. L'article a grandement bénéficié des commentaires des lecteurs anonymes de RAM ainsi que de ceux des professeurs A. Bemmaor et D. Rouziès sur les premières versions de l'article. Cette recherche a été réalisée en partie lors d'un séjour en tant que chercheur visitant à l'Université de Monash (Australie). 
celui-ci d'annoncer précisément l'importance de la réduction de prix (en valeur ou en pourcentage), ni même de rappeler le prix de base du produit. En effet, la valeur réelle de la réduction finale dépendant de la bonne volonté du revendeur, une telle communication pourrait être sanctionnée (publicité mensongère et interdiction des prix imposés).

Le succès, et le budget, d'une opération dépendent de plusieurs facteurs comme l'importance de la réduction de prix, sa répartition entre le détaillant et le client final et les dépenses de la communication associée. La communication de la promotion, ou signal promotionnel, est constituée par tout élément de communication attirant l'attention du client sur l'offre promotionnelle : une affichette ou un autocollant apposé sur le produit portant, par exemple, une mention telle que «promotion », « offre spéciale » ou « offre exceptionnelle ».

Dans une recherche expérimentale, Inman, McAlister et Hoyer (1990) ont montré que ce signal promotionnel a un effet positif sur l'intention d'achat de certains clients. Ils suggèrent alors (p. 79) qu' « un détaillant peut induire un accroissement des ventes... simplement en plaçant un signal promotionnel sur le présentoir de la marque sans vraiment réduire le prix... Le producteur doit moins se préoccuper $d u$ pourcentage de la réduction de prix rétrocédée aux clients que de la mise en place du signal promotionnel sur la marque par les détaillants ».

L'intuition à la base de la recherche présentée ici est que, outre les réserves sur le plan éthique concernant la publicité mensongère, les recommandations d'Inman, McAlister et Hoyer (1990) ne doivent pas être suivies car l'effet positif du signal promotionnel ne peut pas être généralisé. D'abord, cet effet n'a été mis en évidence que pour un traitement superficiel (la promotion attire l'attention mais la valeur économique de la promotion n'est pas analysée), l'effet au niveau des ventes dépend donc de la part des clients ne procédant qu'à ce traitement superficiel. Ensuite, dans le cas inverse d'un traitement approfondi, l'annonce d'une promotion en l'absence d'informations précises sur la réduction peut avoir des effets négatifs par l'influence de cette annonce sur la manière dont le client se représente les alternatives et le choix (cadrage du choix).

La littérature montre que le consommateur évalue l'attractivité d'une offre par rapport à une transaction de référence (Bolton, Warlop et Alba, 2003). Celle-ci est constituée sur la base des informations contextuelles et des informations mémorisées à partir d'expériences antérieures. Ainsi un prix attendu1, que le consommateur s'attend à payer, est élaboré à partir des prix mémorisés (le prix de référence interne de la marque) mais aussi des informations contextuelles comme le prix des concurrents, les informations promotionnelles (Kalwani et alii, 1990) ou même les coûts et profits perçus. D'autres points de référence ont été mis en évidence comme celui de la fréquence des opérations promotionnelles (Lattin et Bucklin, 1989).

Nous formulons l'hypothèse suivante : face à une promotion, le consommateur compare le prix proposé au prix attendu constitué à partir des prix des concurrents et du prix de référence interne de la marque minoré d'un montant correspondant à la réduction promotionnelle de référence. Puisque le rejet d'achat est positivement lié à l'écart entre le prix attendu et le prix proposé, on peut donc avancer la proposition qui suit : tant que la réduction réelle est inférieure à la réduction promotionnelle de référence, le rejet de l'achat est plus important pour un prix annoncé « en promotion » par rapport à un prix normal. Au-delà, l'effet de la réduction de prix se trouve amputé d'un montant considéré comme normal pour une promotion.

Pour que cet effet soit mis en évidence, il convient de réunir plusieurs conditions. La première est que le prix final, réduction comprise, ne doit pas être considéré comme bon marché en valeur absolue. Si le prix annoncé est bon marché, l'effet sera masqué car la promotion sera globalement attractive ; à l'inverse, pour une marque premium dont la réduction de prix serait insuffisante pour rendre la marque attractive par rapport à ses concurrents, l'effet pourrait être négatif sur l'intention d'achat et non nul comme celui observé par Inman, McAlister et Hoyer (1990). La seconde condition est que le prix doit faire l'objet d'un traitement approfondi. L'attention portée au prix est stimulée par la méthodologie avec la demande répétée de choix (acceptation ou rejet de l'achat) pour différents prix (Gabor et Granger, 
1966). La troisième condition est que l'offre promotionnelle n'induise pas un doute sur la qualité, tant en fonction des raisons invoquées pour la promotion (soldes, liquidations ...) que du niveau final atteint par le prix. En effet, dans ce cas, la relation prix-qualité réduirait la valeur du prix attendu pour une qualité perçue inférieure.

Dans un cadre statique, ce comportement est économiquement irrationnel puisque l'offre d'un bénéfice économique réel se traduit par une baisse du taux d'acceptation de l'achat. Plusieurs explications peuvent être avancées, qui correspondent à des motivations spécifiques : (1) une perspective dynamique :si la réduction est inférieure à la réduction habituelle, il peut être plus intéressant pour le consommateur qui sait anticiper les prix de reporter son achat (Kalwani et alii, 1990 ; Sun, Neslin et Srinivasan, 2003) ; (2) un risque sur l'image sociale : si la situation de référence est «en promotion», sans indication du montant de la réduction ou du prix normal de la marque, l'acheteur avisé (smart shopper) n'a pas l'assurance de réaliser une bonne affaire et, dans le doute, peut préférer s'abstenir (Babakus, Tap et Cunningham, 1988) ; (3) une perception accrue de l'iniquité de la transaction : l'incohérence entre l'assimilation de la promotion à une «bonne affaire », d'une part et la perception d'un prix significativement plus élevé que celui des concurrents d'autre part, renforcent la perception du déséquilibre de la transaction (fairness) et peuvent conduire le consommateur à punir la marque trop chère par un refus d'achat (Campbell, 1999).

La recherche diffère de celle d'Inman, McAlister et Hoyer (1990) sur plusieurs points : (1) alors que leur étude concernait à la fois le traitement superficiel et le traitement approfondi du prix, seul ce dernier est étudié ici ; (2) l'intention d'achat avant exposition à la promotion est mesurée, non pas seulement pour la marque considérée, mais pour toutes les marques de l'ensemble évoqué ; (3) le rejet ou l'acceptation d'achat est demandé pour plusieurs prix et non seulement deux (0 ou $15 \%)$; (4) la collecte de données est plus réaliste : les répondants sont des consommateurs en centres commerciaux plutôt que des étudiants. En ce qui concerne les analyses, les intentions d'achat seront traitées comme covariables, analyse effectuée mais non présentée par Inman, McAlister et Hoyer (1990).

L'article comporte quatre parties : les effets promotionnels et leurs origines seront décrits dans la première section, puis le cadre conceptuel sera présenté (section 2), la méthodologie et les résultats seront détaillés (section 3). La discussion des résultats et des limites fera, enfin, l'objet de la quatrième section.

\section{LES EFFETS PROMOTIONNELS ET LEURS ORIGINES}

Les effets promotionnels, mesurés tant au niveau des ventes agrégées qu'à celui des choix individuels, sont à la fois positifs et négatifs.

Sur les ventes, les effets promotionnels à court terme sont généralement positifs (Blattberg et Neslin, 1989). À long terme, certaines techniques peuvent contribuer au développement du marché potentiel (essai) et à la constitution de l'image de marque (primes, jeux). Les effets négatifs à court terme proviennent des comportements d'anticipation et de stockage, alors qu'à long terme ils sont issus de l'accroissement de l'élasticité prix et de la baisse de la rentabilité des investissements publicitaires. Au niveau agrégé, les effets sont difficiles à interpréter car ils résultent à la fois de modifications de comportements et de différences dans la structure de la clientèle.

Au niveau individuel, la promotion influence le choix du consommateur car elle offre directement plusieurs types de bénéfices, utilitaires et hédoniques (Chandon, Wansink et Laurent, 2000). Certains bénéfices peuvent cependant être retournés : l'effet négatif de l'offre d'une prime peu valorisée a été souligné et expliqué par la nécessité de justifier socialement le choix, effet inverse de celui de l' «acheteur avisé » (Simonson et alii, 1994).

En plus du mécanisme promotionnel et de la valeur du bénéfice, l'effet promotionnel dépend de la communication de la promotion (Chen, Monroe et Lou, 1998 ; Inman et McAlister, 1993). La promotion est ainsi une information qui influence le choix, ce qui peut expliquer les effets négatifs à moyen terme sur les ventes (Blattberg et Neslin, 1989). Cette influence est directe par une modification de la qualité perçue (Dodson, Tybout et Sternthal, 1978 ; Raghubir 
et Corfman, 1995) ou indirecte par l'intermédiaire de la relation prix-qualité (Rao et Monroe, 1988). La promotion a aussi un effet sur le prix attendu (Raghubir, 1998) et la sensibilité au prix (Lattin et Bucklin, 1989).

Bien au-delà des seuls bénéfices économiques, la promotion fournit donc d'autres bénéfices qui peuvent justifier une préférence accrue pour la marque en promotion. Ces autres bénéfices, non traités ici, pourraient d'ailleurs expliquer une partie significative des effets promotionnels et justifier la non-observation sur le marché des effets étudiés ici.

\section{CADRE CONCEPTUEL}

Les facteurs qui influencent le plus les choix sont liés aux attributs des marques et aux préférences et expériences d'achat précédentes des consommateurs (Winer, 1986). Si les facteurs liés à la marque (prix et promotion) sont très importants, ils ne peuvent pas cependant dominer l'usage et les préférences (Monroe, 1973 ; Erdem, Mayhew et Sun, 2001). L'usage et les préférences sont donc contrôlés dans l'échantillon.

\section{La comparaison avec le prix attendu}

En vue d'un jugement ou d'un choix, le consommateur construit tout d'abord un cadre de référence (Puto, 1987) comprenant des dimensions comme le prix, la qualité ou la promotion. Pour la dimension prix, l'évaluation est effectuée de manière relative par rapport à un prix attendu (Helson, 1964 ; Monroe, 1973) qui joue un rôle central dans le choix (Kalyanaram et Winer, 1995). Celui-ci est compris, non comme une valeur ponctuelle, mais comme une zone correspondant à des prix acceptables (Zollinger, 1993 ; Kalyanaram et Little, 1995) en dehors de laquelle l'éventualité de l'achat du produit est rejetée.

Les réactions des consommateurs face au prix ne sont pas linéaires et suivent la loi de Weber-Fechner, encore appelée loi de la différence juste perceptible, selon laquelle la perception d'une variation dépend d'un seuil exprimé en pourcentage du niveau actuel du stimulus. Ceci conduit à procéder à un transformation logarithmique des prix (Gabor et Granger, 1966 ; Monroe, 1973 ; Jallais, Orsoni et Fady, 1987).

\section{L'effet de la promotion}

Comment une réduction de prix d'un montant non indiqué est-elle perçue ? Pour un consommateur, la promotion est habituellement associée à un avantage économique. Face à une réduction de prix, il utilise cette information pour inférer le prix inconnu du produit (Raghubir, 1988). En l'absence d'une information précise sur le montant de la réduction, le consommateur va constituer le prix attendu en déduisant du prix de référence interne de la marque le niveau de référence, qu'il estime «normal», pour une réduction de prix (Suri, Manchanda et Kohli, 2000). Par rapport à une situation sans l'annonce de la promotion, le gain perçu par l'écart (prix attenduprix observé) sera donc inférieur ; il pourrait même être négatif. Dans ce cas il devrait, pour un même prix annoncé, réduire le taux d'acceptation (accroître le taux de rejet). L'hypothèse principale testée est donc que, «à prix égal, et lorsque le prix final est supérieur au prix attendu, le taux de rejet d'un produit avec une réduction de prix non précisée est plus élevé que lorsqu'il n'y a pas de promotion annoncée » $(H 1)$.

\section{L'effet du prix}

La zone d'acceptabilité des prix est bordée, en limite inférieure, par un prix trop faible qui fait douter de la qualité et conduit à rejeter l'achat et, en limite supérieure, par un prix jugé trop élevé par rapport à la concurrence et aux ressources du consommateur. Il en résulte une relation non monotone entre l'écart de prix (prix attendu-prix observé) et le rejet de l'achat. Pour éviter les élasticités prix positives des extrêmes, le prix de la marque choisie sera suffisamment élevé et on contrôlera que « le taux de rejet augmente avec le niveau du prix » (H2).

Comme le montrent les études de panels (Blattberg et Neslin, 1989), la communication promotionnelle amplifie l'effet du prix. On suppose alors que, par 
un effet d'interaction du prix et de la promotion, «l'impact positif du prix sur le taux de rejet est supérieur lorsque le produit est en promotion » (H3).

\section{L'effet modérateur de la fidélité}

La prise en compte de l'hétérogénéité individuelle est indispensable pour une bonne estimation des effets des variables (Bell et Lattin, 2000), et il convient d'étudier les facteurs qui peuvent influencer le prix attendu.

Le prix attendu est formé au moment du choix à partir de deux types d'informations : les informations mémorisées sur les prix payés antérieurement et les informations contextuelles disponibles, ce qui conduit à distinguer des prix de référence internes et externes (pour une revue, voir Briesch et alii, 1997 ; Hamelin, 2000 ; Niedrich, Sharma et Wedell, 2001). Le prix de référence interne est souvent opérationnalisé comme un construit spécifique à la marque, par une moyenne pondérée des prix des achats précédents (Briesch et alii, 1997). Les prix contextuels constituent des références externes et, selon la théorie de l'ancrage-ajustement, servent de base à l'élaboration du prix de référence (Monroe, 1973). Puisque l'ordre de présentation des prix influence le prix attendu (Della Bitta et Monroe, 1974), pour une marque premium au prix élevé, l'annonce des prix systématiquement inférieurs des concurrents devrait conduire à une prix attendu situé au-dessous du prix de référence interne.

Le poids des deux informations dépend de facteurs individuels et situationnels. D'après les études sur base de panels (Mayhew et Winer, 1992), les consommateurs recourent simultanément, mais à des degrés divers, aux deux références (Briesch et alii, 1997 ; Mazumdar et Papatla, 2000). L'élaboration dépend de la volonté du consommateur de faire l'effort d'un traitement cognitif des informations. Même si des variables individuelles comme le besoin d'intellectualiser (Inman, McAlister et Hoyer, 1990) peuvent entrer en compte, la méthodologie utilisée devrait induire un traitement approfondi pour tous les répondants car elle conduit à des demandes répétées de jugement avec une seule variable modifiée : le prix.

Les informations contextuelles, saillantes et facilement accessibles, ont souvent une forte importance dans la constitution du prix de référence ; elles sont pri-traitement approfondi des informations sur le prix, en

vilégiées pour un produit à faible fréquence d'achat (Mazumdar et Papatla, 2000). Au contraire, l'utilisation des prix mémorisés dépend de leur accessibilité et de leur pertinence ; elle est influencée par des facteurs individuels comme le nombre de marques achetées, les préférences ou la fidélité à la marque (Rajendran et Tellis, 1994). Si les prix de référence internes sont implicites et ne peuvent être directement mesurés, on peut cependant supposer que, selon le raisonnement précédent, les acheteurs habituels d'une marque accordent une importance relative plus forte aux prix de référence internes qu'aux prix contextuels. En outre, s'il s'agit d'une marque au prix élevé, le prix de référence interne sera plus élevé. Ainsi, « un acheteur habituel de la marque a un taux de rejet plus faible pour un prix donné » (H4).

Une relation négative entre le niveau de prix des produits achetés et la sensibilité au prix a été mise en évidence (Gabor et Granger, 1966 ; Sinha et Batra, 1999 ; Bell et Lattin, 2000). On s'attend donc à ce que, par l'interaction entre prix et marque, «l'effet positif du prix sur le taux de rejet (soit) plus faible pour les acheteurs habituels de la marque »(H5). Les acheteurs habituels de la marque connaissant mieux le prix de celle-ci, l'écart (prix attendu-prix observé) devrait être perçu plus clairement et donc, par l'interaction entre promotion et marque, «l'effet positif de l'annonce en promotion sur le taux de rejet est plus faible pour les acheteurs habituels de la marque » (H6).

Il est difficile d'anticiper l'effet de l'interaction triple entre le prix, la promotion et l'achat habituel de la marque. Une meilleure connaissance du prix habituel de la marque permet aux acheteurs habituels de procéder à des achats d'anticipation lors des promotions et l'on peut avancer de manière exploratoire que «l'effet modérateur de la promotion sur l'effet positif du prix sur le taux de rejet de l'achat est amplifié pour les acheteurs habituels de la marque »(H7).

\section{MÉTHODOLOGIE}

\author{
e
}

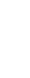
La méthodologie utilisée cherche à induire un 
vue de l'acceptation ou du rejet de l'achat d'une marque à prix élevé dont la qualité du produit n'est pas mise en doute. Les données concernent un produit de grande consommation, les bâtonnets de crème glacée vendus par boîte de trois. Trois marques sont présentées au consommateur, la marque premium A qui fait l'objet de l'étude et dont le prix variera, et deux autres marques dont les prix restent stables : une seconde marque nationale $\mathrm{B}$ et une marque de distributeur $\mathrm{C}$.

\section{Les variables collectées}

Les données sont collectées selon la procédure de Gabor et Granger (1966) qui consiste à demander l'acceptation ou le rejet de l'achat, et dans ce dernier cas, la raison du refus, pour des prix tirés au hasard parmi dix prix espacés de manière identique (voir, en Annexe, les questions posées). L'entretien comporte différentes séquences : (1) le recueil des marques de l'ensemble évoqué et des intentions d'achat de trois marques (A, B, C) sans rappel des prix, mesurées sur une échelle de 1 à 7 (IAk ); (2) l'annonce des prix des produits concurrents en euros $(1,65$ pour la marque de distributeur $\mathrm{C}, 2,15$ pour la marque nationale B) ; (3) le recueil des mesures binaires d'acceptation/ rejet pour la marque étudiée (A) pour chacun des 10 prix ( IAk ). Ceux-ci, espacés de $0,1 €$, vont de 2,0 à $2,9 €$ pour un prix effectif de la marque de $2,4 €$ et subissent une transformation logarithmique. Du fait de l'introduction récente de l'euro, des convertisseurs ont été proposés. L'acception d'achat est une variable bivalente (oui/non) complétée par la raison du rejet (trop cher - trop bon marché). La variable dépendante, le rejet d'achat, est une variable binaire (Rj ) qui vaut 1 si l'achat de la marque A est refusé parce que le prix ( pj ) est trop cher (= 0 sinon). Le Ta bleau 1 reprend la description des variables. Le plan expérimental inter-sujets $(2 \times 2)$ croise l'achat habituel de la marque et la manipulation « en promotion ». Deux groupes (MARQUE) sont constitués selon que la marque A est la marque achetée le plus fréquemment ou non. Pour chaque groupe, deux sous-groupes de répondants reçoivent aléatoirement un questionnaire « prix normal » ou « en promotion ». La manipulation consiste, pour le groupe en promotion seulement, à annoncer et indiquer sur la photographie du produit la mention «en promotion » (PROMO).

Avec un effectif de 80 dans chaque case, l'échantillon est composé de 320 personnes interrogées en face-à-face par des enquêteurs professionnels dans les centres commerciaux de cinq grandes villes de France (Paris, Lille, Nantes, Toulouse, Marseille) en août 2002 (VILLE). Les répondants sont des acheteurs de la catégorie de produit en GMS, recrutés sur quotas d'âge (25-34, 35-49 et 50-60 ans), le critère de l'appartenance de la marque $\mathrm{A}$ à leur ensemble évoqué et sur la marque achetée le plus fréquemment (marque A ou une autre). La structure de l'échantillon est présentée dans le Tableau 2 et on peut relever une erreur de quotas pour le groupe à prix normal avec deux questionnaires en plus pour les acheteurs de la marque A aux dépens des acheteurs des autres marques. La base de données comporte 3200 observations $(80 \times 2 \times 2 \times 10)$.

Tableau 1. - Récapitulatif des variables

\begin{tabular}{|l|l|}
\hline VILLE Cinq & villes où ont été réalisés les entretiens (Paris, Lille, Nantes, Toulouse, Marseille) \\
\hline ÂGE Trois & ranches d'âge (25-34, 35-49 et 50-60 ans) \\
\hline PROMO Młntion « En promotion » sur la marque étudiée $(A)$ (variable centrée sur 0) codée $=0,5$ en promotion, \\
sinon
\end{tabular}


Tableau 2. - Structure de l'échantillon

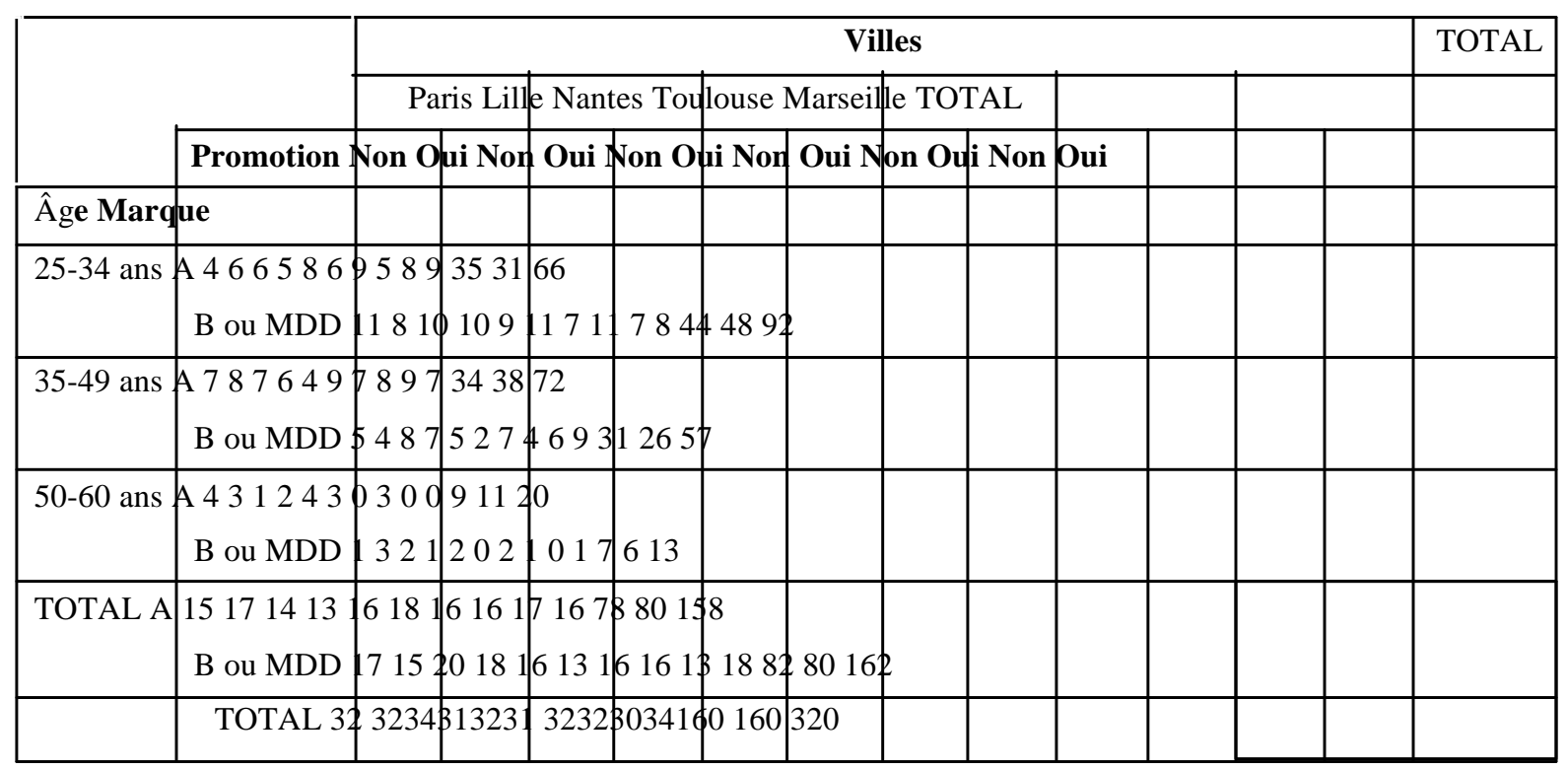

\section{Le modèle}

Les réponses des consommateurs sont analysées dans le cadre d'un modèle logit binomial, comme cela a déjà été effectué sur ce type de données (Wedel et Leeflang, 1998). La partie déterministe individuelle du modèle de rejet $\mathrm{d}$ ' achat du produit $\mathrm{A}$ au prix j par un consommateur $(\mathrm{Vj})$ s'écrit alors :

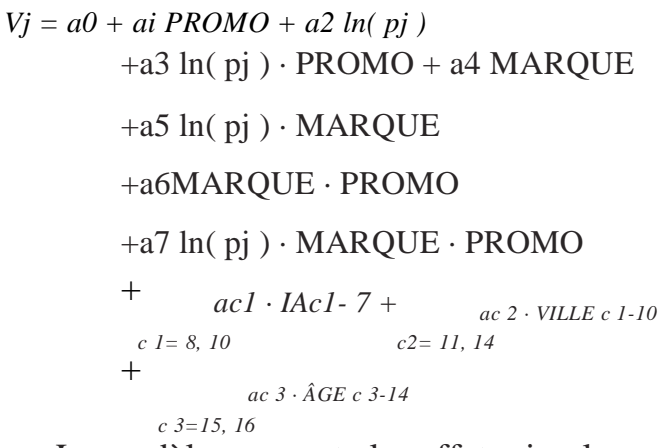

Le modèle comporte les effets simples pour Ville, $\hat{A} \mathrm{ge}$ et Intentions d'achat et les effets simples et les interactions d'ordre 1 et 2 entre les variables Marque et Promotion et 1 à 3 entre ces deux variables et le Prix. L'analyse d'un tel modèle est complexe et, sur suggestion d'un lecteur anonyme, les recommandations d'Irwin et McClelland (2001) ont été suivies : centrage des variables en fonction des objectifs pour- suivis et maintien de toutes les interactions d'ordre inférieur, même non significatives, lorsqu'une interaction d'ordre supérieur est significative.

\section{RÉSULTATS STATISTIQUES}

\section{Analyses descriptives}

Dans le traitement «en promotion », les taux de rejet sont systématiquement supérieurs au contrôle quel que soit le groupe analysé, comme le montrent les Figures 1 et 2 .

Le taux de rejet sur l'échantillon est de $44 \%$ avec une différence significative selon le traitement en promotion (49\%) versus prix normal (39\%), et ce, quel que soit le groupe : marque A préférée (35\% versus $27 \%$ ) ou autres marques préférées (63\% versus $51 \%$ ). Le Tableau 3 présente les pourcentages de rejet selon les groupes (marque $*$ promotion). Les $a$ écarts entre les taux de rejet en promotion ou non sont presque systématiquement négatifs. La différence est significative à un risque bilatéral de $1 \%$ avec le test de Kolmogorov-Smirnov. 


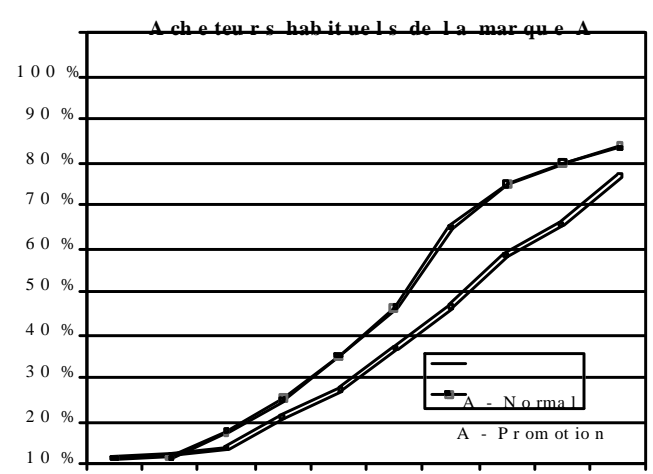

$0 \%$

2, $02,12,22,32,42,52,62,72,82,9$

Figure 1. - Taux de rêjet (Trop cher)

pour les acheteurs habituels de la marque $\mathrm{A}$

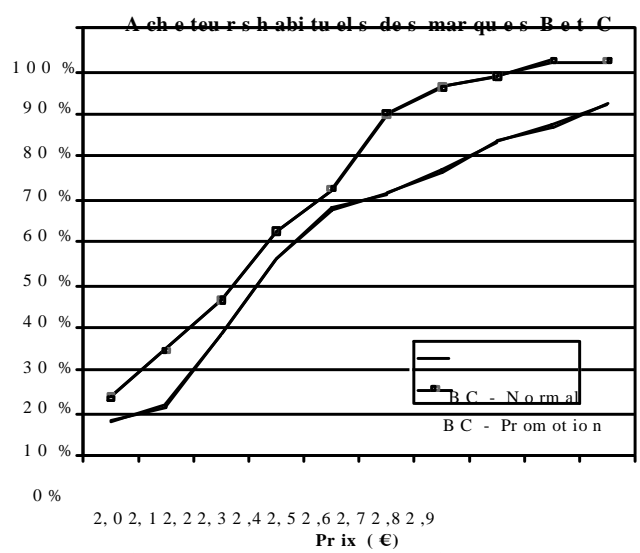

Figure 2. - Taux de rejet (Trop cher) pour les acheteurs habituels des marques B et $\mathrm{C}$

Tableau 3. - Pourcentages de rejet d'achat de la marque A selon le prix,

l'annonce en promotion et l'achat habituel de la marque

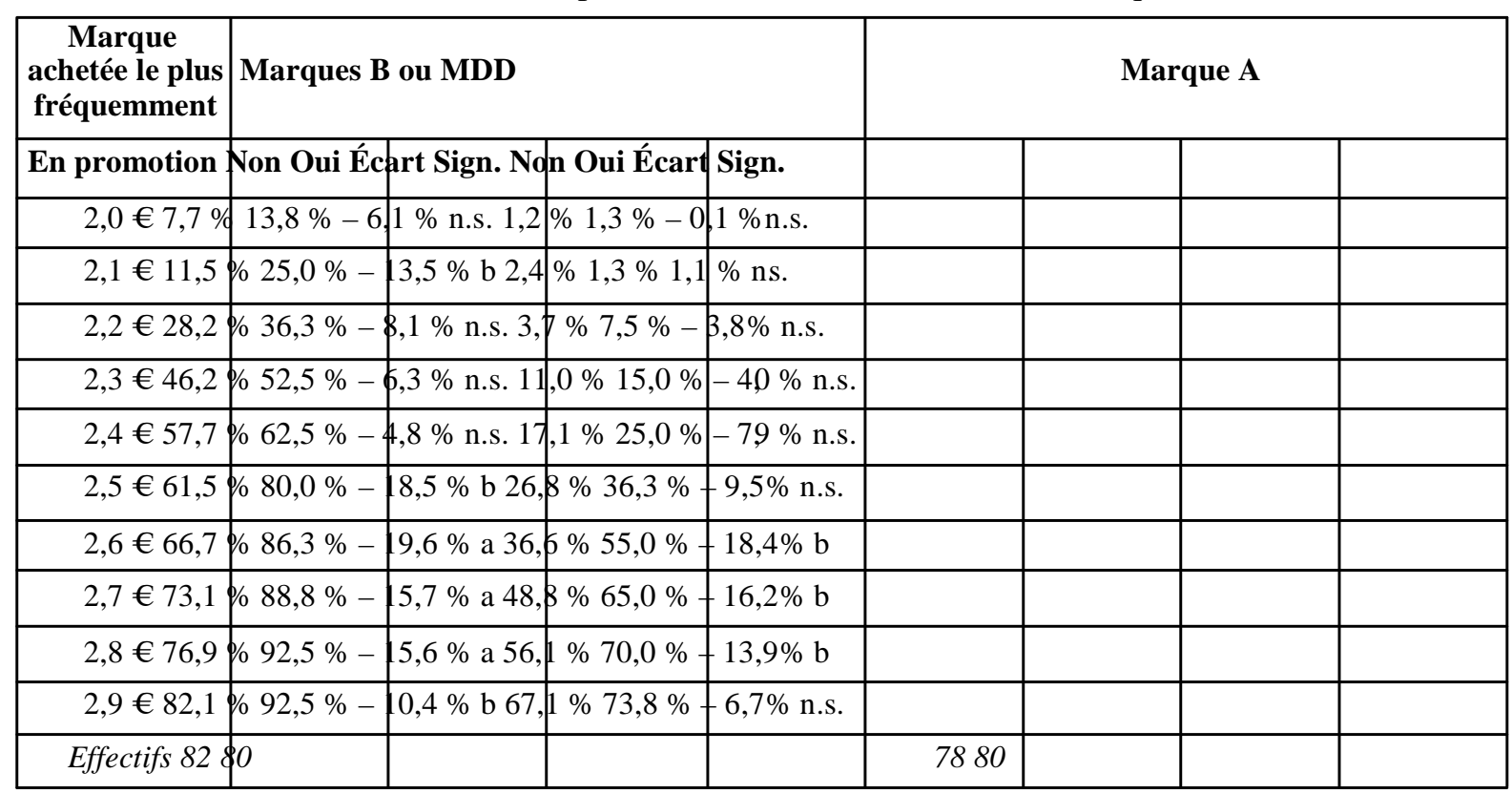

Chi2 test : sign $\cdot a<0,01, b<0,05, c<0,10$

Des tests du chi2 sur chaque niveau de prix montrent que, au niveau agrégé, l'effet ne devient systématiquement significatif qu'au-delà du niveau de prix habituel du produit $(2,4 €)$. Ceci peut s'expliquer par la taille trop réduite de l'échantillon pour détecter des différences lorsque les taux de rejet sont faibles. À ce prix, le taux de rejet par les acheteurs habituels est non négligeable $(17 \%)$, cet effet étant probablement lié65 \% obtenus par Gabor et Granger sur plusieurs pro- à l'annonce préalable des prix, plus faibles, des concurrents (Della Bitta et Monroe, 1974). Au-delà de ce prix, le taux de rejet n'augmente que graduellement, montrant ainsi que les répondants n'ont pas une idée précise du prix habituel de la marque (LeBoutillier, LeBoutillier et Neslin, 1994). Au prix normal de la marque, le taux d'acceptation est de $63 \%$, proche des 65 \% obtenus par Gabor et Granger sur plusieurs pro- 
duits. La taille moyenne de l'ensemble évoqué est de 1,93 marques avec $30 \%$ répondants n'ayant que la marque A et $23 \%$ ayant les trois marques.

\section{Résultats des estimations}

Les paramètres du modèle sont estimés par la procédure LOGISTIC de SAS. La qualité globale de l'ajustement est évaluée par la statistique U2 (dont l'interprétation est similaire à celle du R2 en régression) qui rapporte l'amélioration de la vraisemblance à celle d'un modèle nul ne comportant qu'une constante. Les résultats figurent dans le Tableau 4 avec les coefficients et leurs écarts types ainsi que la significativité selon le test du chi2.

Par rapport au modèle nul et au modèle 1, ne comportant que les constantes liées à l'échantillon, la

Tableau 4. - Paramètres estimés

Modèle 1 Modèle 2 Modèle 3

\section{Modèle Nul (constante seule) 4 388,49}

Vraisemblance (- 2 Log Likelihood) 4 230,14 2 857,61 2 764,85

$\mathrm{U} 2$

$158,351530,881623,64$

$0,0360,3490,370$

Coefficient Sign. Coefficient Sign. Coefficient Sign.

Constante (coefficient) a $0-0,1596$ a - 0,5348 a 1,0650 a

(écart type) 0,458 0,0643 0,2691

\begin{tabular}{|c|c|c|c|}
\hline Promo & $a 1$ & $\begin{array}{l}0,6001 \text { a } 0,6924 \text { a } \\
0,10870,1111\end{array}$ & \\
\hline $\ln ($ Prix $)$ & $a 2$ & $\begin{array}{l}13,6303 \text { a } 14,1613 \text { a } \\
0,51900,5385\end{array}$ & \\
\hline $\ln ($ Prix $) *$ Promo & $a 3$ & $\begin{array}{l}2,1565 \mathrm{~b} 2,3267 \mathrm{~b} \\
0,99551,0127\end{array}$ & \\
\hline Marque & $a 4$ & $\begin{array}{l}-1,8333 \mathrm{a}-1,1697 \mathrm{a} \\
0,11120,1347\end{array}$ & \\
\hline $\ln ($ Prix $) *$ Marque a5 & & $\begin{array}{l}1,8482 \mathrm{c} 1,7704 \mathrm{c} \\
0,99661,0136\end{array}$ & \\
\hline Promo*Marque & $a 6$ & $\begin{array}{l}-0,3875 \mathrm{c}-0,4961 \mathrm{~b} \\
0,21780,2221\end{array}$ & \\
\hline $\ln ($ Prix $) *$ Promo*Marque a7 & & $\begin{array}{l}-1,8478 \text { n.s. }-2,1147 \text { n.s. } \\
1,99022,0233\end{array}$ & \\
\hline $\mathrm{IA}(\mathrm{A})$ & $a 8$ & & $\begin{array}{l}-0,3403 \mathrm{a} \\
0,0434\end{array}$ \\
\hline $\mathrm{IA}(\mathrm{B})$ & $a 9$ & & $\begin{array}{l}0,0497 \mathrm{a} \\
0,0179\end{array}$ \\
\hline $\mathrm{IA}(\mathrm{C})$ & $a 10$ & & $\begin{array}{l}0,1151 \mathrm{a} \\
0,0201\end{array}$ \\
\hline Ville_1 & $\begin{array}{r}a 110,1850 \text { b } 0,2926 \text { a } 0,1278 \text { n.s. } \\
0,07200,09250,1070\end{array}$ & & \\
\hline Ville_2 & $\begin{array}{r}a 120,0218 \text { n.s. } 0,1860 \text { b 0,2781 a } \\
0,07160,09170,0946\end{array}$ & & \\
\hline Ville_3 & $\begin{array}{r}a 13-0,7807 a-1,3301 a-1,5131 a \\
0,07980,10360,1167\end{array}$ & & \\
\hline Ville_4 & $\begin{array}{c}\text { a14 - 0,0156 n.s. - 0,0446 n.s. }-0,0535 \text { n.s. } \\
0,07210,09170,0949\end{array}$ & & \\
\hline Age_1 & $\begin{array}{r}a 15-0,2506 a-0,1975 a-0,1827 b \\
0,05500,07080,0736\end{array}$ & & \\
\hline Age_2 & $\begin{array}{c}a 16-0,0244 \text { n.s. }-0,1028 \text { n.s. }-0,1435 c \\
0,05690,07260,0742\end{array}$ & & \\
\hline
\end{tabular}


prise en compte des variables prix, promotion et marque (modèle 2) accroît significativement la qualité de l'ajustement global $(\mathrm{U} 2=0,349$ versus $\mathrm{U} 2=$ 0,036). Celle-ci est encore améliorée par la prise en compte des intentions d'achat des marques (modèle $3, \mathrm{U} 2=0,370)$.

Le signe positif du coefficient de la promotion (a1 >0) pour la promotion est cohérent avec l'hypothèse que l'annonce « en promotion » accrôt le taux de rejet (H1). Le prix a bien un effet positif sur le taux de rejet $(\mathrm{a} 2>0, \mathrm{H} 2)$ et l'interaction significative et positive prix * promotion $(\mathrm{a} 3>0, \mathrm{H} 3)$ permet de conclure à un effet plus important du prix sur le rejet d'achat quand la marque est en promotion.

Le coefficient négatif (a4) indique que l'achat habituel de la marque A réduit le taux de rejet, ce qui était attendu (H4). En outre, le rôle modérateur de l'achat habituel de la marque est bien mis en évidence. Ainsi l'interaction marque * promotion est négative et significative (a6) et l'effet de l'annonce en promotion sur le rejet est plus faible pour les acheteurs de la marque A (H6). En revanche, l'interaction prix $*$ marque $($ a $5>0$ ) n'est que marginalement significative. De même, l'hypothèse d'un effet plus important du prix en promotion pour les acheteurs de la marque (H7) ne peut pas être acceptée car le coefficient négatif de l'interaction prix * promotion * marque (a7) n'est pas significatif. Les coefficients (a8, a9 et a10 ) des variables individuelles d'intention d'achat sont statistiquement significatifs et ont le signe attendu : une intention d'achat plus forte de la marque $\mathrm{A}(\mathrm{a} 8>0)$ réduit le rejet de la marque à un prix donné, alors que celle de la marque nationale $\mathrm{B}$ $(a 9>0)$ ou de distributeur $C(a 10>0)$ l'accroît.

\section{DISCUSSION ET LIMITES}

Le consommateur est convaincu que la promotion est généralement intéressante, au point d'en faire un critère de choix prépondérant lorsqu'il ne souhaite pas effectuer une analyse approfondie de la situation. C'est l'effet du signal promotionnel mis en évidence par Inman, McAlister et Hoyer (1990) pour des écarts de prix faibles entre les concurrents, unique- ment lorsque la situation a fait l'objet d'un traitement superficiel. L'objectif de l'étude est d'explorer uniquement le cas complémentaire d'un traitement approfondi des données par le répondant, pour lequel Inman, McAlister et Hoyer (1990) n'ont pas obtenu d'effet significatif lorsque le signal promotionnel n'était pas accompagné d'une réduction effective du prix. Si l'effet signal ne joue que positivement dans le cas d'un traitement superficiel (effet d'attraction de l'attention, effets non monétaires), dans le cas d'un traitement approfondi, des valeurs de référence (prix et promotion) sont aussi retrouvées en mémoire, et elles influencent, conjointement avec les prix contextuels, le prix attendu. La résultante sur le choix peut donc éventuellement être négative.

Dans ce cadre nous avons postulé que la réduction promotionnelle du prix, lorsqu'elle n'est pas précisée, réduit le prix de référence interne d'un montant qui correspond à la valeur mémorisée d'une réduction habituelle. Mais le prix attendu est aussi influencé par les prix contextuels et, si la réduction de prix transmise au consommateur par le distributeur est insuffisante pour faire apparaître un bénéfice économique pour une marque au prix élevé, alors le signal promotionnel aura un effet négatif sur l'acceptation d'achat. En cas d'incohérence entre le signal promotionnel (habituellement associé à un avantage monétaire) et la perception d'un écart de prix positif entre le prix attendu et le prix affiché, la valeur accordée au signal promotionnel devient négative.

Cette hypothèse a été corroborée dans l'expérimentation menée et ce résultat tend donc à souligner que l'effet du signal promotionnel, sans mention de la réduction de prix et lorsque le consommateur procède à un examen approfondi de l'offre, n'est pas toujours positif ou nul. Si l'on y ajoute l'effet négatif dû à une réduction potentielle de la qualité perçue, ceci souligne la nécessité, pour l'annonceur d'une promotion, de communiquer (le prix de base ou le montant de la réduction en valeur ou en pourcentage, les raisons de la promotion) afin de réduire les inférences effectuées par le client sur les points de référence du choix (qualité, prix, promotion). L'effet modérateur de la fidélité à la marque est aussi mis en évidence : l'achat habituel de la marque renforce le prix de référence interne et rend le consommateur moins sensible aux prix contextuels, ce qui a pour effet de réduire l'importance de l'effet du signal promotionnel. 
Sur le plan théorique, ce résultat s'inscrit dans la lignée des conclusions récentes sur l'importance de l'effet de la promotion sur le plan informatif (Raghub et Corfman, 1999), même pour une promotion par réduction de prix. Au-delà des effets économiques immédiats, la promotion, en influençant le cadre du choix, peut avoir un effet immédiat négatif, si elle est perçue comme insuffisante par rapport aux pratiques antérieures, et un effet différé négatif par la réduction du prix attendu qu'elle entraîne. Ces résultats confirment les études précédentes, mettant en évidence qu'au-delà des bénéfices utilitaires et hédoniques, la promotion a une dimension informationnelle importante; en l'absence des informations nécessaires, celle-ci contribue à l'élaboration du cadre de la décision en influençant les autres dimensions du choix comme la qualité ou le prix, ainsi que leurs importances respectives.

Sur le plan managérial, ce résultat signifie qu'en l'absence d'information sur le prix de base ou le montant de la réduction offerte, une partie de celle-ci est annulée lorsque le client procède à une analyse approfondie de l'offre. Dans le cadre d'une baisse de prix de $15 \%$, le fait de ne pas annoncer le montant de la réduction conduit le consommateur animé d'une intention d'achat moyenne (4 sur 7) à défalquer un montant d'environ $6,3 \%$ pour obtenir la réduction réelle perçue. Cette constatation pourrait être une explication à l'observation de la faible efficacité des réductions de prix de faibles montants (Leeflang et alii, 2000).

Cette observation entraîne deux recommandations. La première consiste à souligner l'importance de l'information fournie lors de la communication de la promotion. Non seulement celle-ci doit justifier la promotion, pour éviter l'inférence d'une qualité inférieure, mais elle devrait aussi indiquer soit le prix de référence à utiliser, soit le montant de la réduction pratiquée pour éviter que le consommateur ne fasse des inférences.

Pour une opération promotionnelle, il convient de procéder à un arbitrage budgétaire entre le montant de la réduction de prix et le budget de communication de l'opération. Lorsque l'entreprise prend en charge la gestion des relations avec les clients (coupons, offres de remboursement), elle maîtrise le montant offert et peut le communiquer mais les frais de traitement sont élevés. L'octroi au revendeur d'une remise, qu'il transmet éventuellement et en partie au client final, est une solution moins coûteuse mais les résultats obtenus ici montrent qu'une partie de ir la réduction est déjà effacée par la modification des attentes des clients sur le prix. L'importance de ce budget gaspillé rend tout à fait rentable la recherche d'un meilleur contrôle de la réduction effectivement pratiquée.

Plusieurs limites doivent être prises en compte. La première est que les observations ne concernent que le cas d'une marque premium dont le prix est significativement supérieur à celui des concurrents. Les prix contextuels des concurrents sont alors systématiquement inférieurs au prix de référence interne de la marque et ont tendance, par effet d'ancrage, à réduire le prix attendu. Un prix moins élevé conduirait à un prix promotionnel perçu comme significativement inférieur aux prix des concurrents et réduirait, voire annulerait, l'effet mesuré ici. La seconde limite est liée à la méthodologie choisie, la méthode de Gabor et Granger. L' attention accrue portée à la variable modifiée (le prix) limite la validité externe du coefficient et surestime l'effet prix ; celui-ci doit être calé par des informations sur les élasticités de la demande au prix obtenues avec des panels distributeurs (Wildner, 1988). Ainsi, pour une réduction de prix de $15 \%$ et une intention d'achat moyenne (4 sur 7), l'élasticité prix est évaluée à - 2,97 pour les acheteurs de la marque et à - 6,20 pour les non-acheteurs, ce qui est assez élevé par rapport aux résultats des méta-analyses (Tellis, 1988). Le choix de proposer des prix supérieurs au prix habituel de la marque peut aussi avoir influencé les réponses des consommateurs qui connaissent le prix habituel de la marque et se sentent manipulés. Enfin, le cadre expérimental choisi a conduit à surpondérer les acheteurs de la marque et à déformer l'échantillon puisque tous les répondants doivent avoir la marque $\mathrm{A}$ dans leur ensemble évoqué.

\section{RÉFÉRENCES BIBLIOGRAPHIQUES}

Babakus E., Tat P. et Cunningham W. (1988), Coupon redemption: a motivational perspective, The Journal of Consumer Marketing, 5,2,37-43.

Bell D. et Lattin J. (2000), Looking for loss aversion in scanner panel data: the confounding effect of price response heterogeneity, Marketing Science, 19, 2, 185-200. 
Blattberg R. et Neslin S. (1989), Sales promotion: the long and the short of it, Marketing Letters, 1,1,81-97.

Briesch R., Krisnamurthi L., Mazumdar T. et Raj S.P. (1997) A comparative analysis of reference price models, JournalLeef of Consumer Research, 24, 2, 202-214.

Bolton L., Warlop L. et Alba J. (2003), Consumer perceptions of price (un)fairness, Journal of Consumer Research, 19, 1, 474-491.

Campbell M. (1999), Perceptions of price unfairness: antecedents and consequences, Journal of Marketing Research, 36, 2, 187-199.

Chandon P., Wansink B. et Laurent G. (2000), A benefit congruency framework of sales promotion effectiveness, Journal of Marketing, 64, 4, 65-81.

Chen S., Monroe K.B. et Lou Y. (1998), The effects of framing price promotion messages on consumers' perceptions and purchase intentions, Journal of Retailing, 74, 3, 353-372.

Della Bitta A. et Monroe K.B. (1974), The influence of adaptation level on selective price perceptions, Advances in Consumer Research,1,éds. S. Ward et $P$. Wright, Provo, Utah, Association for Consumer Research, 359-369.

Erdem T., Mayhew G. et Sun B. (2001), Understanding reference-price shoppers: a within and cross-category analysis, Journal of Marketing Research, 38, 4, 445-457.

Gabor A. et Granger C.W.J. (1966), Price as an indicator of quality: report on an inquiry, Economica, 33, 129, 43-70.

Guadagni P. et Little J. (1983), A logit model of brand choice calibrated on scanner data, Marketing Science, $2,3,203-238$.

Hamelin J. (2000), Le prix de référence : un concept polymorphe, Recherche et Applications en Marketing, 15, $3,75-88$

Helson H. (1964), Adaptation-level theory,New York, Harper \& Row.

Inman J. et McAlister L. (1993), A retailer promotion model considering promotion signal sensitivity, Marketing Science, 12, 4, 339-356.

Inman J., McAlister L. et Hoyer W. (1990), Promotion signal: proxy for a price cut?, Journal of Consumer Research, 17, 3, 74-81.

Irwin J. et McClelland G. (2001), Misleading heuristics and moderated multiple regression models, Journal of Marketing Research, 38, 1, 100-109.

Jallais J., Orsoni J. et Fady A. (1987), Marketing de la distribution, Vuibert, Paris.

Kalwani M.U., Yim Ch.K., Rinne H. et Sugita Y (1990), A price expectations model of customer brand choice, Journal of Marketing Research, 27, 3, 251-262.

Kalyanaram G. et Little J.D.C. (1995), An empirical analysis of latitude of price acceptance in consumer package goods, Journal of Consumer Research, 21, 3, 408-418.

Kalyanaram G. et Winer R.S. (1995), Empirical generalizations from reference price and asymmetric price response $Z$ research, Marketing Science, 14, 3, G161-G169.

Lattin J. et Bucklin R. (1989), Reference effects of price and promotion on brand choice behavior, Journal of Marketing Research, 26, 3, 299-310.
LeBoutillier J., LeBoutillier S. et Neslin S. (1994), A replication and extension of the Dickson and Sawyer priceawareness study, Marketing Letters,5,1,31-42.

eeflang P., Wittink D., Wedel M. et Naert P. (2000), Building models for marketing decisions, International series in quantitative marketing, Kluwer, Dordrecht.

Mayhew G. et Winer R.S. (1992), An empirical analysis of internal and external reference price effects using scanner data, Journal of Consumer Research, 19, 1, 62-70.

Mazumdar T. et Papatla P. (2000), An investigation of reference price segments, Journal of Marketing Research, $37,2,246-258$.

Monroe K.B. (1973), Buyers' subjective perceptions of price, Journal of Marketing Research, 10, 1, 70-80.

Niedrich R., Sharma S. et Wedell D. (2001), Reference price and price perceptions: a comparison of alternative models, Journal of Consumer Research, 28, 3, 339-354.

Puto C. (1987), The framing of buying decisions, Journal of Consumer Research, 14, 3, 301-315.

Rajendran K. et Tellis G. (1994), Contextual and temporal components of reference price, Journal of Marketing, $58,1,22-34$.

Raghubir P. (1998), Coupon value: a signal for price?, Journal of Marketing Research, 35, 3, 316-324.

Raghubir P. et Corfman K. (1999), When do price promotions affect pretrial brand evaluations?, Journal of Marketing Research, 36, 2, 211-222.

Simonson I., Carmon Z. et O'Curry S. (1994), Experimental evidence on the negative effect of product features and sales promotions on brand choice, Marketing Science, 13, 1, 23-39.

Sun B., Neslin S. et Srinivasan K. (2003), Measuring the impact of promotions on brand switching when consumers are forward looking, Journal of Marketing Research, 40, 4, 389-405.

Suri R. et Monroe K.B. (2001), The effects of need for cognition and trait anxiety on price acceptability, Psychology \& Marketing, 18, 1, 21-42.

Suri R., Manchanda R. et Kohli C. (2000), Brand evaluations: comparison of fixed price and discounted price offers, Journal of Product \& Brand Management,9,3,193-206.

Tellis G. (1988), The price elasticity of selective demand: a meta-analysis of econometric models of sales, Journal of Marketing Research, 25, 4, 331-341.

Wedel M. et Leeflang P.S.H. (1998), A model for the effects of psychological pricing in Gabor-Granger price studies, Journal of Economic Psychology, 19, 2, 237-260.

Wildner R. (1998), The introduction of the Euro: the importance of understanding consumers' reactions, Actes du 5le Congrès de l'ESOMAR, The power of

Winer R.S. (1986), A reference price model of brand choice for frequently purchased products, Journal of Consumer Research, 13, 2, 250-256.

Zollinger M. (1993), Le concept de prix de référence dans le comportement du consommateur : d'une revue de la littérature à l'élaboration d'un modèle prix de référence-acceptabilité, Recherche et Applications en Marketing, 8,2,61-77. 


\section{ANNEXE}

\section{Questions posées}

Ensemble évoqué :«Quelles sont toutes les

marques de batônnets que vous achetez, ne serait-ce que de temps en temps?»

Intention d'achat :[Présenter les 3 produits et l'échelle de réponse] "Vous allez m'indiquer, à

l'aide de cette échelle, votre intention d'achat pour chacun de ces produits, sans tenir compte de la variété. »

Acceptation-rejet : Nous allons maintenant parler d'une boîte de (A) [en promotion]. Le prix de (B) est de 2,15 €. Le prix de (C) est de 1,65 €. Voici une enveloppe, tirez un papier. Si cette boîte de (A) était vendue au prix de (prix tiré), l'achèteriez-vous?

Sinon, est-ce parce que vous la trouvez trop chère ou trop bon marché ?» 
